\title{
Comparison of the Prophylactic Effect of Silymarin and Deferoxamine on Iron Overload-Induced Hepatotoxicity in Rat
}

\author{
Hossein Najafzadeh • Mohammad Razi Jalali • \\ Hassan Morovvati • Farnaz Taravati
}

Published online: 25 February 2010

(C) American College of Medical Toxicology 2010

\begin{abstract}
In pathologic conditions or poisoning states, iron overload can affect different tissues including liver. In this study, the prophylactic effect of deferoxamine and silymarin was compared in decreasing experimental ironoverload-induced hepatotoxicity in rats. The study was done in six groups of rats, which received drugs q2 days for 2 weeks. The rats in groups 1 to 6 received drugs, respectively: normal saline, iron dextran, iron dextran+deferoxamine (intraperitoneally), iron dextran+silymarin (orally), iron dextran+ silymarin (intraperitoneally), and iron dextran + deferoxamine (intraperitoneally)+silymarin (intraperitoneally). At the end of the study, blood was collected, and serum was separated for laboratory tests. The liver of rats was separated for iron measuring and tissue processing. The serum iron concentration and the serum alanine aminotransferase (ALT) and aspartate aminotransferase (AST) activity were determined. The numbers of necrotic hepatocytes were counted as quantity index tissue injury in light microscopic examination. The mean of serum and liver iron in group 2 was significantly greater than group 1. Liver iron was significantly decreased in other groups except group 4 . Also serum iron was decreased in groups 3 to 6 compared to group 2 (nearly 400\%). ALT activity in group 3 and AST activity in group 5 were significantly lesser than in other groups. The mean of necrotic hepatocytes in group 2 was significantly increased in comparison to group 1. This elevation was significantly prevented by deferoxamine and silymarin. The result of the present study shows that silymarin has a protective effect similar to deferoxamine on iron overload-induced hepatotoxicity.
\end{abstract}

H. Najafzadeh $(\varangle) \cdot$ M. Razi Jalali $\cdot$ H. Morovvati $\cdot$ F. Taravati Shahid Chamran University,

Ahvaz, Iran

e-mail: najafzadeh@scu.ac.ir

e-mail: najafzadehvarzi@yahoo.com
Keywords Silymarin · Deferoxamine · Iron overload · Rats · Hepatotoxicity

\section{Introduction}

Iron overload in iron poisoning and in some diseases causes several disorders that related to oxidative stress and depression of immune system. The iron overload is a relatively common finding in many patients with a variety of end-stage liver diseases [4, 22].

The pathogenesis of hepatic fibrosis in patients with iron overload is not well understood. But four possible mechanisms have been suggested. Firstly, iron is as an inducer of fibrosis even in the absence of necrosis and inflammation and may act as a profibrogenic agent that stimulates the deposition of collagen. Secondly, iron, as a mediator of hepatocellular necrosis and local inflammation, may activate the peroxidative process and produce oxygen-free radicals, lipid peroxidation damage to protein, and DNA and stimulation of fibrosis [10, 11, 31]. Thirdly, iron, as an inducer of fibrosis, may act in conjunction with other hepatotoxins [24, 30]. It has been suggested that mild to moderate hepatic iron concentration may exacerbate liver injury and accelerate the development of hepatic fibrosis [3, 13]. Fourthly, iron overload may alter hepatic extracellular matrix degradation [14]. In addition, excessive iron deposition in liver will lead to further injury such as hepatocellular necrosis, inflammation, fibrosis, and in some cases even carcinoma [39].

Clinically, deferoxamine (desferal) is the most current drug to decrease concentration of iron in patients. This drug is injected, which is uncomfortable for patients. In addition, this agent chelates iron and some microelements and has side effect on the ear and bone. So, it is ideal to find better agent for iron overload treatment especially in thalasemic patients [20]. 
Silymarin, an antioxidant flavonoid complex derived from the herb milk thistle (Silybum marianum) has long been used in the treatment of liver diseases [12, 21, 27, 33]. This property seems to be due to its ability to scavenge free radicals and to chelate metal ions $[6,8]$. It is a scavenger of radicals, such as hydroxyl, superoxide, and hydrogen peroxide $\left(\mathrm{H}_{2} \mathrm{O}_{2}\right)$, and increases SOD and decreases lipid peroxidation $[28,37]$. The silymarin is capable of protecting liver cells directly by stabilizing the membrane permeability through inhibiting lipid peroxidation $[9,15$, 26] and preventing liver glutathione depletion [35]. In addition, there are a number of other effects of silymarin at the cellular and molecular level, such as alteration of DNA expression via suppression of nuclear factor [7]. Soto et al. reported that the protective effect of silymarin on pancreatic damage induced by alloxan may be due to an increase in the activity of antioxidant enzymes such as superoxide dismutase and glutathione peroxidase [34].

Thus, the aims of the present study were to evaluate the prophylactic effect of iron toxicity on liver tissue and compare the effect of silymarin and deferoxamine to ameliorate the hepatotoxicity of excessive iron because deferoxamine is iron chelator and silymarin also had iron chelatory effect in vitro.

\section{Materials and Methods}

\section{Materials}

Silymarin was purchased from Sigma Co. Iron dextran was purchased from Sterop Co., Belgium. Desferal was purchased from Novartis Co., Switzerland. Commercial kit for alanine aminotransferase (ALT) and aspartate aminotransferase (AST) measurement was purchased from Pars Azmon Co., Iran.

\section{Animals}

Adult male Wistar rats, weighing 180-220 g, were obtained from the Animal Center of the University of Jondishpour. The animals were kept under standard conditions and had access to a standard diet and clean drinking water.

\section{Methods}

The animals were divided at random into six groups of six animals each. The rats received drugs q2 days for 2 weeks. The first group received saline intraperitoneally (IP), and this group served as control. The second group received iron dextran $100 \mathrm{mg} / \mathrm{kg}$ IP. The third group received iron dextran $100 \mathrm{mg} / \mathrm{kg}$ IP with deferoxamine at a dose of $50 \mathrm{mg} / \mathrm{kg}$ IP. The fourth group received iron dextran
$100 \mathrm{mg} / \mathrm{kg}$ IP with silymarin $200 \mathrm{mg} / \mathrm{kg}$ orally. The silymarin was prepared in ethanol (as vehicle). The fifth group received iron dextran $100 \mathrm{mg} / \mathrm{kg}$ IP with silymarin $200 \mathrm{mg} / \mathrm{kg}$ IP. The sixth group received iron dextran $100 \mathrm{mg} / \mathrm{kg}$ IP with deferoxamine at a dose of $50 \mathrm{mg} / \mathrm{kg}$ IP and silymarin $200 \mathrm{mg} / \mathrm{kg}$ IP. The deferoxamine or silymarin was injected at different protean sites (right and left).

After 14 days, the animals were anesthetized, and blood samples were obtained. The serum samples were separated for the measurement of serum ALT and AST levels that were estimated according to the method of commercial kits. These enzymes can detect liver injury, especially AST, which is more specific for liver of rats. The concentration of iron in serum and liver was measured with an autoanalyzer apparatus (Eppendorf Co., Germany).

Sections from the liver of each animal were fixed in phosphate-buffered formaldehyde and embedded in paraffin, and 5- $\mu \mathrm{m}$-thick sections were prepared. The sections were stained with hematoxylin and eosin for the evaluation of liver tissue. The necrotic hepatocytes were counted in five microscopic fields, and the mean of these cells was determined at each group.

Data were expressed as mean \pm SEM. Group variance was analyzed by one-way analysis of variation, and Fisher least significant difference test was tested for significant differences between groups. $P \leq 0.05$ was considered statistically significant.

\section{Results}

Administration of iron dextran $100 \mathrm{mg} / \mathrm{kg}$ q2 days for 14 days resulted in a significant increase in serum and liver iron concentrations. The mean of serum iron concentration

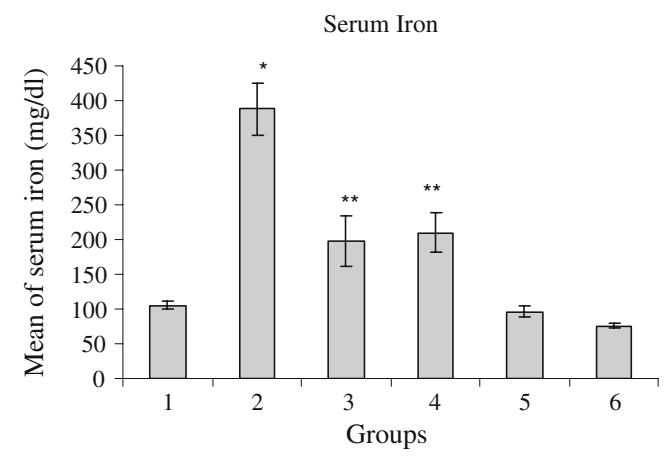

Fig. 1 Serum iron concentration (mean $\pm \mathrm{SEM}$ ) in rats $(n=6)$ (single asterisk significantly different from control group, double asterisks significantly different from iron dextran group; level of significance, 0.05). The rats in groups 1 to 6 received the following drugs, respectively: normal saline, iron dextran, iron dextran + deferoxamine (intraperitoneally), iron dextran+silymarin (orally), iron dextran+ silymarin (intraperitoneally), and iron dextran + deferoxamine (intraperitoneally)+silymarin (intraperitoneally) 


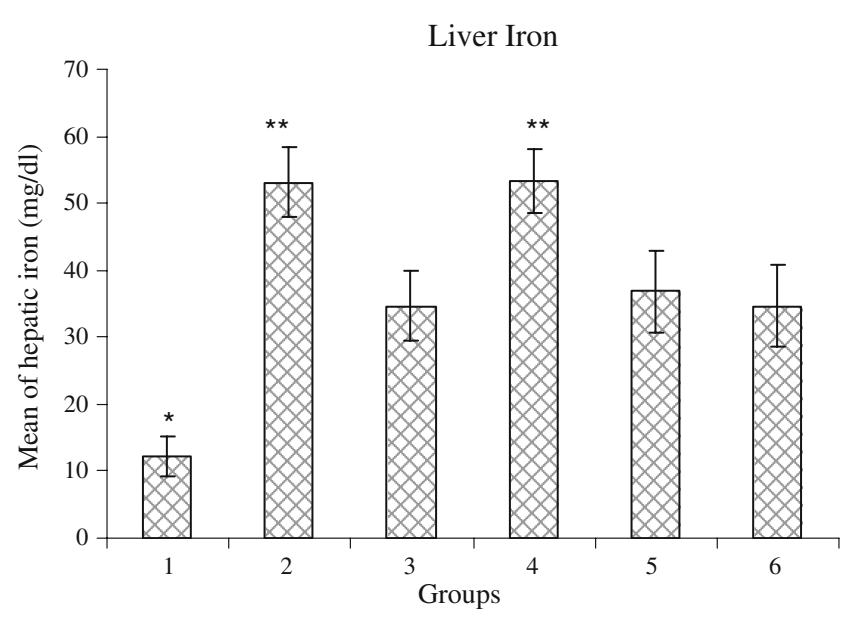

Fig. 2 Liver iron concentration (mean \pm SEM) in rats $(n=6)$ (single asterisk significantly different from other groups, double asterisks significantly different from groups $1,3,5$, and 6 ; level of significance, $0.05)$. The rats in groups 1 to 6 received the following drugs, respectively: normal saline, iron dextran, iron dextran+deferoxamine (intraperitoneally), iron dextran+silymarin (orally), iron dextran+ silymarin (intraperitoneally), and iron dextran+deferoxamine (intraperitoneally) + silymarin (intraperitoneally)

in group $2(387.6 \mathrm{mg} / \mathrm{dl})$ was significantly greater than in group $1(105.12 \mathrm{mg} / \mathrm{dl})(p<0.001)$. The concentration of serum iron was significantly decreased in group 3 (received iron dextran and deferoxamine) in comparison to group 2 $(p=0.003)$. This mean was significantly different in group 4 (which received iron dextran and orally administered silymarin) $(p=0.008)$, and in groups 5 (received iron

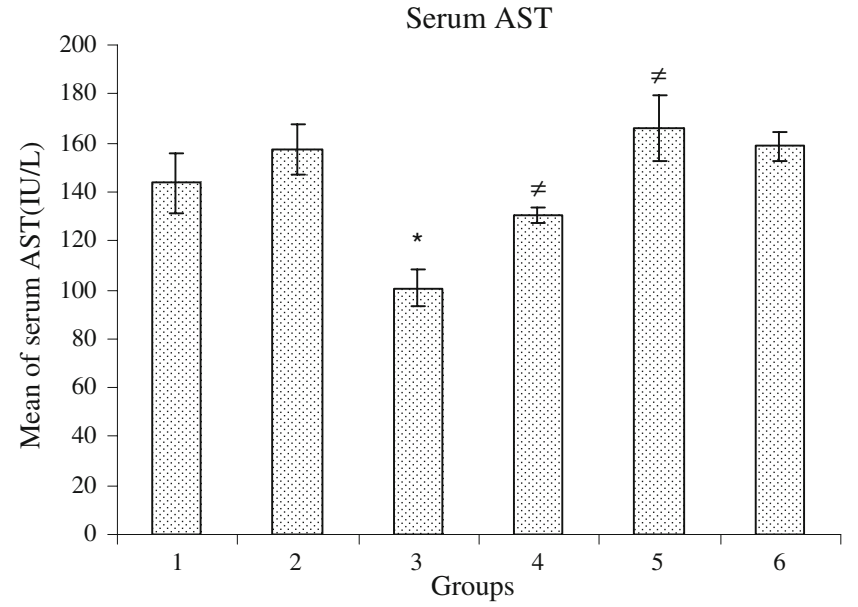

Fig. 4 Serum AST concentration (mean $\pm \mathrm{SEM}$ ) in rats $(n=6)$ (single asterisk significantly different from other groups, numbers signs significantly different from iron dextran group; level of significance, 0.05). The rats in groups 1 to 6 received the following drugs, respectively: normal saline, iron dextran, iron dextran + deferoxamine (intraperitoneally), iron dextran+silymarin (orally), iron dextran+ silymarin (intraperitoneally), and iron dextran + deferoxamine (intraperitoneally)+silymarin (intraperitoneally)

dextran and silymarin) $(p=0.001)$ and 6 (which received iron dextran and silymarin and deferoxamine $)(p<0.001)$ in comparison to group 2 (Fig. 1). The mean of liver iron concentration in group $2(53.1 \mathrm{mg} / \mathrm{dl})$ was significantly greater than group $1(12.16 \mathrm{mg} / \mathrm{dl})(p<0.001)$. Also, liver iron level was significantly decreased in other groups except group 4 in comparison to group 2 (Fig. 2).

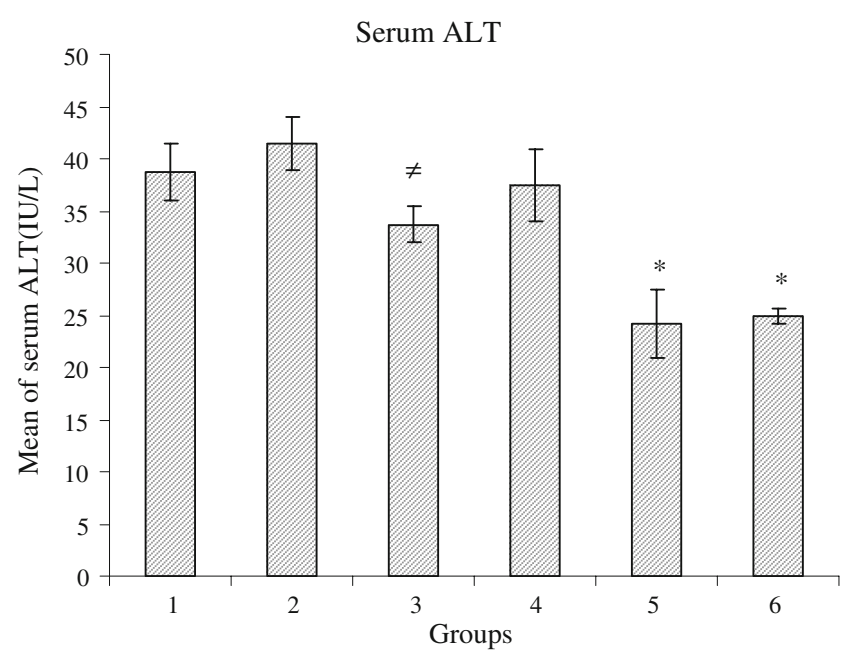

Fig. 3 Serum ALT concentration (mean \pm SEM) in rats $(n=6)$ (single asterisks significantly different from other groups, number sign significantly different from iron dextran group; level of significance, 0.05). The rats in groups 1 to 6 received the following drugs, respectively: normal saline, iron dextran, iron dextran + deferoxamine (intraperitoneally), iron dextran+silymarin (orally), iron dextran+ silymarin (intraperitoneally), and iron dextran + deferoxamine (intraperitoneally) + silymarin (intraperitoneally)

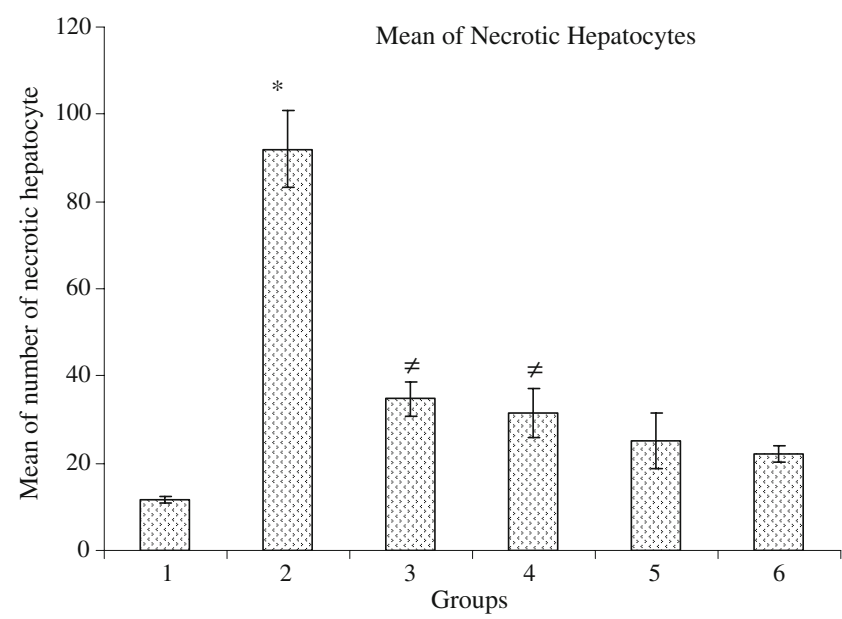

Fig. 5 Number of necrotic cells (mean \pm SEM) in rats $(n=6)$ (single asterisk significantly different from other groups, number signs significantly different from control group; level of significance, 0.05). The rats in group 1 to 6 received the following drugs, respectively: normal saline, iron dextran, iron dextran+deferoxamine (intraperitoneally), iron dextran+silymarin (orally), iron dextran+ silymarin (intraperitoneally), and iron dextran+deferoxamine (intraperitoneally)+silymarin (intraperitoneally) 
The serum activity of ALT in group 2 was increased, but this increase was not significant in comparison to group 1 . This mean was significant in groups $3(p=0.04)$ and $5(p=$ 0.001 ), but not in group 4 in comparison to group 2 (Fig. 3). Serum activity of AST in group 2 was increased, but this increase was not significant in comparison to group 1. The activity of this enzyme in group 3 was significantly lesser than other groups $(p<0.005)$, and its mean in group 4 was decreased in comparison to group 5 (Fig. 4).

The mean number of necrotic hepatocytes in group 2 was significantly increased in comparison to group 1 , and was greater than nine times compared to group $1(p<$ $0.001)$. This mean was significantly decreased in other groups in comparison to group $2(p<0.001)$ (Fig. 5).

\section{Discussion}

The role of iron in the progression of hepatic damage in various clinical and experimental conditions has usually been studied by iron loading [16, 23, 29].

In the present study, iron dextran induced hepatotoxicity as manifested by an increase in the number of necrotic hepatocytes in histopathological examination compared to control animals. The number of necrotic hepatocytes was counted based on morphological changes in light microscope; thus the organelle injury may not be parallel to morphological changes. The results of present study is similar those previously reported [5, 38, 39].

We demonstrated that silymarin and deferoxamine have a protective effect on iron-induced hepatotoxicity in rats. Similarly, other researchers mentioned this finding. Ahmed et al. reported that silymarin has an antihepatotoxic activity against carbon tetrachloride-induced hepatotoxicity in rats. The silymarin protects liver against increase in serum ALT, AST, and alkaline phosphates and decrease in total protein and total albumin [2]. The deferoxamine has antioxidative effect along with iron chelatory property [18].

Also, silymarin is known to have hepatoprotective and anticarcinogenic effects $[19,25]$. The silymarin can chelate ferrous iron. This chelation can raise the activity to the level of most active scavengers, possibly by site-specific scavenging [1]. The silymarin can also inhibit lipid peroxidation by reacting with peroxy radicals. This ability of silymarin leads to a significant increase in the cellular antioxidant defense machinery by ameliorating the deleterious effects of free radical reaction and the increase in GSH content, which is important in maintaining the ferrous state [1, 32].

In the other study, the effects of increasing dietary levels of Fe on the histopathology of liver, pancreas, spleen, and heart were examined in a rat model for iron overload. Sprague-Dawley rats were fed diets containing 35, 350, 3,500 , or $20,000 \mu \mathrm{g} \mathrm{Fe} / \mathrm{g}$, and, after 12 weeks, there was a direct correlation between increased liver nonheme Fe and lipid peroxidation. Histopathological examination of tissues revealed hepatocellular hemosiderosis in all groups of rats [38]. The protective effect of baicalin (a nature flavonoid) on liver of iron overload mouse may be due to both the antioxidant and iron chelation activities of baicalin [39].

Jensen and et al. investigated the relationship between the extent of hepatocellular injury as reflected by serum levels of ALT and AST and several iron status indices in 39 anti-hepatitis $\mathrm{C}$ virus-negative patients with transfusional iron overload owing to acquired anemia. They observed that serum levels of ALT and AST were directly involved in iron toxicity [17].

In our previous study, administration of silymarin caused a generally protective and ameliorative effect against gentamicin-induced nephrotoxicity in dogs [36]. The protective effect of silymarin is associated with its antioxidant properties, as it possibly acts as a free radical scavenger, lipid peroxidation inhibitor, and preservation of the activity of total serum antioxidants [17]. The prophylactic effect of silymarin and deferoxamine on iron overload-induced nephrotoxicity in rats was evaluated by our research team. The results show that silymarin had a beneficial effect on decreasing tubular necrosis induced by iron in rats.

In summary, the result of present study shows that silymarin has a protective effect similar to deferoxamine on controlling serum iron concentration and liver injury, which is induced by iron overload. It seems that this efficacy of silymarin can be evaluated in clinical trials.

Acknowledgment The authors wish to express their gratitude to the research council of Shahid Chamran University for their financial support of present study.

\section{References}

1. Abu Ghadeer AR, Ali SE, Osman SA, Abu Bedair FA, Abbady MM, El-Kady MR (2001) Antagonistic role of silymarin against cardiotoxicity and impaired antioxidant induced by adriamycin and/or radiation exposure in albino rats. Pakistan J Biol Sci 4:604-607

2. Ahmed B, Khan SA, Alam T (2003) Synthesis and antihepatotoxic activity of some heterocyclic compounds containing the 1 , 4-dioxane ring system. Pharmazie 58:173-176

3. Arezzini B, Lunghi B, Lungarella G, Gardi C (2003) Iron overload enhances the development of experimental liver cirrhosis in mice. Int J Biochem Cell Biol 35:486-495

4. Bacon BR, Britton RS (1990) The pathology of hepatic iron overload: a free radical-mediated process? Hepatol 11:127-137

5. Bhattacharya A, Ramanathan M, Ghosal S, Bhattacharya SK (2000) Effect of withania samra glycoeithanolides on iron-induced hepatotoxicity in rats. Phytother Res 14:568-570

6. Borsari M, Gabbi C, Ghelfi F, Grandi R, Saladini M, Severi S, Borella F (2001) Silybin, a new iron-chelating agent. J Inorg Biochem 85:123-129 
7. Chon SK, Kim NS (2005) Evaluation of silymarin in the treatment on asymptomatic Giardia infections in dogs. Parasitol Res 97:445-451

8. Crocenzi FA, Sanchez Pozzi EJ, Pellegrino JM, Rodriguez Garay EA, Mottino AD, Roma MG (2003) Preventive effect of silymarin against taurolithocholate-induced cholestasis in the rat. Biochem Pharmacol 66:355-364

9. Dong JJ, Bauer M (2001) Antifibrotic effect of silymarin in rat secondary biliary fibrosis is mediated by down regulation of procollagen1 (I) and TIMP-1. J Hepatol 35:392-398

10. Emerit J, Beaumont C, Trivin F (2001) Iron metabolism, free radicals and oxidative injury. Biomed Pharmacother 55:333-339

11. Farinati F, Cardin R, De Maria N, Della Libera G, Marafin C, Lecis E, Burra P, Floreani A, Cecchetto A, Naccarato R (1995) Iron storage, lipid peroxidation and glutathione turnover in chronic anti-HCV positive hepatitis. J Hepatol 22:449-456

12. Flora K, Hahn M, Rosen H, Benner K (1998) Milk thistle (Silybum marianum) for the therapy of liver disease. Am J Gastroenterol 93(2):139-43

13. George DK, Goldwurm S, MacDonald GA, Cowley LL, Walker NI, Ward PJ, Jazwinska EC, Powell LW (1998) Increased hepatic iron concentration in nonalcoholic steatohepatitis is associated with increased fibrosis. Gastroenterol 114:311-318

14. George DK, Ramm GA, Powell LW, Fletcher LM, Walker NI, Cowley LL, Crawford DH (1998) Evidence for altered hepatic matrix degradation in genetic haemochromatosis. Gut 42:715720

15. Hakova H, Misurova E, Kropacova R (1996) The effect of silymarin on the concentration and total content of nucleic acids in tissues of continuously irradiated rats. Vet Med (Praha) 41:113119

16. Houglum K, Filip M, Josephl W, Chojkier M (1990) Malondialehyde and 4-hydroxyno nenal protein adducts in plasma and liver of rats with iron overloads. J Clinic Investig Inc 86:1991-1998

17. Jensen PD, Jensen FT, Christensen T, Nielsen JL, Ellegaard J (2003) Relationship between hepatocellular injury and transfusional iron overload prior to and during iron chelation with desferrioxamine: a study in adult patients with acquired anemias. Blood 101(1):91-96

18. Kadikoylu G, Bolaman Z, Demir S, Balkaya M, Akalin N, Enli Y (2004) The effects of desferrioxamine on cisplatininduced lipid peroxidation and the activities of antioxidant enzymes in rat kidneys. Hum Exp Toxicol 23:29-34

19. Kang JS, Jeon YJ, Park SK, Yang KH, Kim HM (2004) Protection against lipopolysaccharide-induced sepsis and inhibition of interleukin-1 beta and prostaglandin E2 synthesis by silymarin. Biochem Pharmacol 67:175-181

20. Kontoghiorghes GJ, Pattichi K, Hadjigavriel M, Kolnagou A (2000) Transfusional iron overload and chelation therapy with deferoxamine and deferiprone $\left(\mathrm{L}_{1}\right)$. Transfus Sci 23:211-223

21. Laekeman G, De Coster S, De Meyer K, Leuven KU (2003) St. Mary's Thistle: an overview. J Pharm Belg 58:28-31
22. Ludwig J, Hashimoto E, Porayko MK, Moyer TP, Baldus WP (1997) Hemosiderosis in cirrhosis: a study of 447 native livers. Gastroenterol 112:882-888

23. Mayers M, Brent G, Prendergast F, Holman R, Kuntz S, Larusso N (1991) Alterations in the structure, physiochemical properties, and $\mathrm{pH}$ of hepatocyte lysosomes in experimental iron overload. J Clin Invest Inc 88:1207-1215

24. Medina J, Moreno-Otero R (2005) Pathophysiological basis for antioxidant therapy in chronic liver disease. Drugs 65:2445-2461

25. Mereish KA, Bunner DL, Regland DR, Creasia DA (1991) Protection against microcystin-L. R. induced hepatotoxicity by silymarin: biochemistry, histopathology and lethality. Pharmacol Res 8:257-277

26. Mira L, Silva M, Manso CF (1994) Scavenging of reactive oxygen species by silibin dihemisuccinate. Biochem Pharmacol 48:753-759

27. Naveau S (2001) Acute alcoholic hepatitis treatments. Press Med 30:1024-1030

28. Oliveira C, Lopasso FP, Laurindo F, Leitao R, Laundanna AA (2001) Protection against liver ischemia-reperfusion injury in rats by silymarin or verapamil. Transplant Proc 33:3010-3014

29. Peretz G, Link G, Pappo O, Bruck R, Ackerman Z (2006) Effect of hepatic iron concentration reduction on hepatic fibrosis and damage in rats with cholestatic liver disease. World J Gastroenterol 12(2):240-245

30. Pietrangelo A (1996) Metals, oxidative stress, and hepatic fibrogenesis. Semin Liver Dis 16:13-30

31. Poli G, Parola M (1997) Oxidative damage and fibrogenesis. Free Radic Biol Med 22:287-305

32. Ramadan L, Roushdy HM, Abu Senna GM, Amin NE, El-Deshw OA (2002) Radioprotectve effect of silymarin against radiation induced hepatotoxicity. Pharmacol Res 45:447-452

33. Saller R, Meier R, Brignoli R (2001) The use of silymarin in the treatment of liver disease. Drugs 61(4):2034-2061

34. Soto C, Recoba R, Barron H, Alvarez C, Favari L (2003) Silymarin increases antioxidant enzymes in alloxan-induced diabetes in rat pancreas. Comp Biochem Physiol C Toxicol Pharmacol 136:205-212

35. Valenzuela A, Monica A, Soledad V, Ricardo G (1989) Selectivity of silymarin on the increase of the glutathione content in different tissues of the rats. Planta Med 55:420-422

36. Varzi NH, Esmailzadeh S, Morovvati H, Avizeh R, Shahriari A, Givi EM (2007) Effect of silymarin and vitamin E on gentamicininduced nephrotoxicity in dogs. J Vet Pharmacol Ther 30:477-481

37. Vladimir K, Daniela W (2005) Silybin and silymarin-new effects and applications. Biomedical Papers 149:29-41

38. Whittaker P, Hines FA, Robl MG, Dunkel VC (1996) Histopathological evaluation of liver, pancreas, spleen, and heart from ironoverloaded Sprague-Dawley rats. Toxicol Pathol 24(5):558-563

39. Zhao Y, Li H, Gao Z, Xu H (2005) Effects of dietary baicalin supplementation on iron overload-induced mouse liver oxidative injury. Europ J Pharmacol 509(2-3):195-200 